\title{
Desarrollo de un Software de Apoyo el Desarrollo de la Función Ejecutiva en Niños con Trastornos del Espectro Autista
}

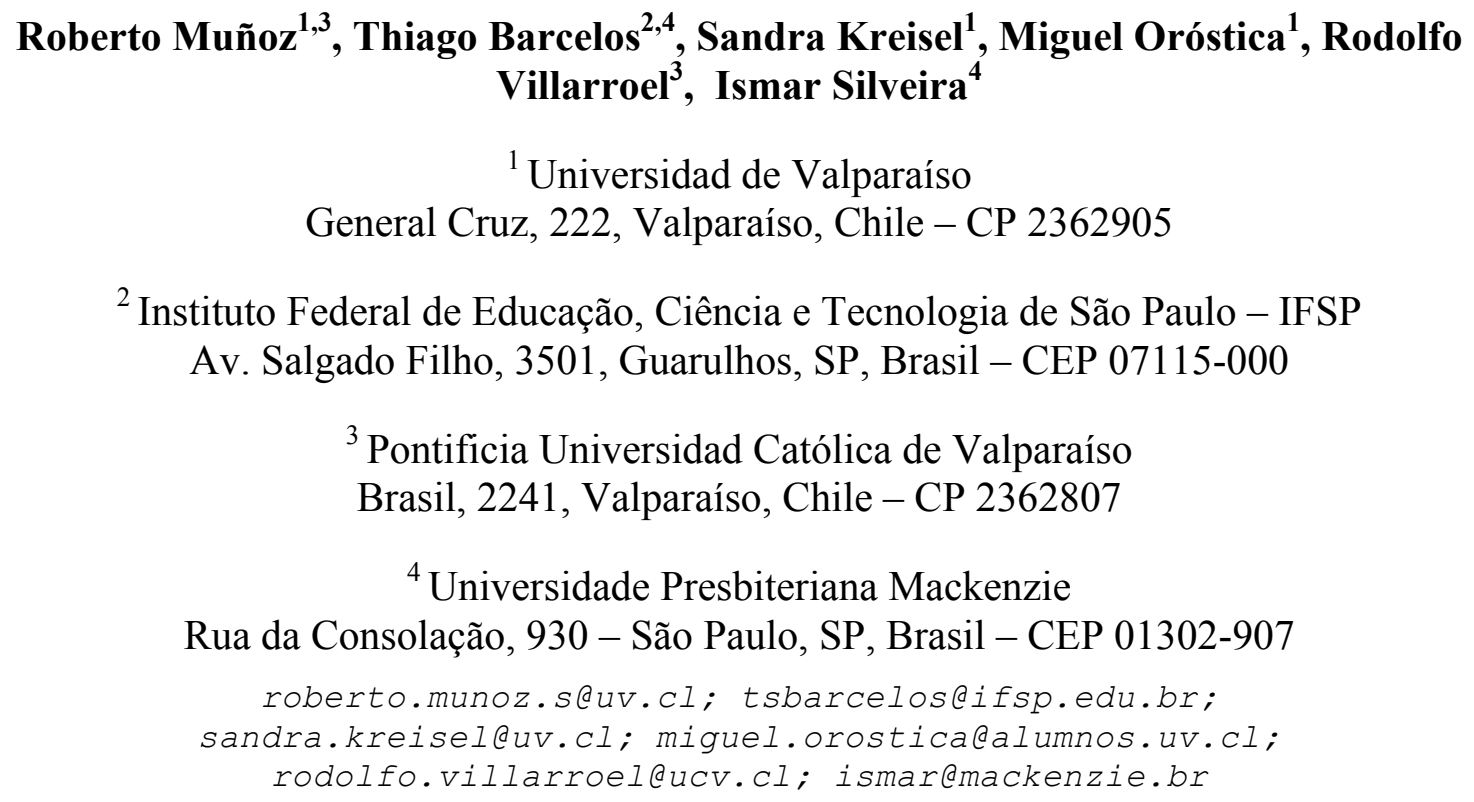

\begin{abstract}
The Autism Spectrum Disorder is a neurodevelopment condition characterized by a decrease in social interaction, communication, creativity, imagination and organization of daily activities of those who have this condition. The executive function is a set of mental processes that helps connecting past experience with present action. Currently in Chile, there are no solutions that support development or improvement of these cognitive skills in autistic children by taking advantage of features present in multi-touch devices. For this reason, this study describes the development of software that supports the improvement of the executive function in children with autism spectrum disorder. The developed application, surpasses 10,000 installations in the Google Play platform.
\end{abstract}

Resumen. Los Trastornos del Espectro Autista (TEA) son una condición del neurodesarrollo caracterizada por una disminución en la interacción social, comunicación, creatividad, imaginación y organización de las actividades diarias de aquellos que tienen esta condición. La función ejecutiva es un conjunto de procesos mentales que ayudan a conectar las experiencias pasadas con una acción presente. Actualmente en Chile, no existe soluciones que apoyen o mejoren estas habilidades cognitivas en niños con TEA, tomando como ventaja las características de los dispositivos multitouch. Por tal razón, este trabajo describe el proceso de desarrollo de una aplicación que apoya el desarrollo de la función ejecutiva en niños con TEA. La aplicación propuesta, sobrepasa las 10.000 instalaciones en la plataforma Play de Google. 
CBIE-LACLO 2015

Anais dos Workshops do IV Congresso Brasileiro de Informática na Educação (CBIE 2015)

\section{Introducción}

El trastorno del espectro autista (TEA) es un condición del neurodesarrollo que principalmente se caracteriza por una disminución en la interacción social, comunicación, creatividad, imaginación y la organización de las actividades de la vida diaria de las personas que poseen esta condición (AITKEN, 2011). Hoy en día, el número de niños diagnosticados con TEA, sigue creciendo, lo que nos recuerda de las necesidades de esta comunidad (MUNOZ et al., 2012). En la mayoría de los casos, las características de la persona con TEA persisten por el resto de su vida, sin embargo, las personas que poseen este trastorno pueden mejorar su condición con el apoyo de equipos multidisciplinarios (VOLKMAR; REICHOW; WIESNER, 2011).

La función ejecutiva es el constructo cognitivo usado para describir conductas dirigidas hacia una meta, orientadas hacia el futuro, que se consideran mediadas por los lóbulos frontales. Éstas incluyen la planificación, inhibición de respuestas prepotentes, flexibilidad, búsqueda organizada y memoria de trabajo (OZONOFF et al., 1994), por lo que su déficit implica una serie de problemas a quienes poseen TEA (VAN DEURZEN et al., 2008).

Asimismo las herramientas tecnológicas pueden ser de gran ayuda para el abordaje del TEA, siempre y cuando éstas sean complementarias (DIGENNARO REED; HYMAN; HIRST, 2011). Las Tecnologías de la Información y la Comunicación (TICs), dado el gran avance que han tenido en los últimos años, se han ido transformando en un instrumento de apoyo muy aceptado dentro del abordaje terapéutico (DIGENNARO REED; HYMAN; HIRST, 2011). Si bien el progreso de las herramientas automatizadas o sistemas que ayuden al desarrollo de las habilidades existen alrededor del mundo (BEREZNAK et al., 2012), (MECHLING; GAST; SEID, 2009), (HOURCADE et al., 2013), en Chile no se pueden utilizar de la mejor manera, ya que factores como el idioma e idiosincrasia propias de cada país, entorpecen en gran medida la tarea que se busca desarrollar.

Por las razones antes expuestas, se desarrolló una aplicación para dispositivos multitouch que tuvo como fin el dar un soporte a los profesionales de apoyo (fonoaudiólogos, educadores diferenciales, psicólogos) que abordan las habilidades cognitivas en niños/as con TEA, centrándose en la función ejecutiva. Para esto, fue necesario trabajar en instituciones educacionales donde se pudieron visualizar las necesidades de los niños con TEA, y corroborar la usabilidad e interacción de la aplicación con el usuario final de los dispositivos multitouch.

El resultado, en cuanto a su funcionalidad, fue un software que posee 3 niveles de actividades para los distintos ámbitos dentro de la vida de los niños y niñas con TEA, estos ámbitos son casa, escuela y calle, cada uno de ellas posee distintas actividades que están relacionadas con la organización y planificación en la vida de los niños y niñas. Por otra parte, la aplicación propuesta, a agosto del 2015, posee sobre 11.000 descargas en la plataforma de difusión masiva Play Google ("Proyect@Habilidades - Aplicaciones de Android en Google Play", 2015).

\section{Problemática Actual}

En la actualidad las TICs, en el ámbito local, escasamente forman parte de las estrategias terapéuticas para el fomento de las habilidades cognitivas de los pacientes 
con TEA, principalmente debido a la falta de herramientas desarrolladas para el público Chileno. Esto conlleva el inconveniente de que las aplicaciones que han sido generadas fuera del ámbito nacional han sido desarrolladas para ser utilizadas por el usuario de las regiones donde son llevabas a cabo, representado sus culturas y costumbres, las que en la mayoría de los casos son muy distintas a nuestra idiosincrasia, lo que afecta cuando se trata de recibir servicios y/o terapias adecuadas en el ambiente local.

\section{Metodología de Desarrollo}

Para el desarrollo de este trabajo se utilizó la metodología de Diseño Centrado en el Usuario, esto debido a que es una metodología ampliamente aceptada para el diseño de aplicaciones de software que puedan utilizarse y que realmente satisfagan de las necesidades de sus usuarios (VAN DIJK et al., 2011). En las secciones siguientes se describe cómo cada etapa de la metodología se contextualizó a este trabajo.

\subsection{Fase 1: Definición de usuarios objetivo y requisitos}

Para este proyecto fue seleccionado un grupo de niños con TEA entre 8 e 11 años, que asisten a una escuela especial Chilena, profesores, terapeutas y padres, con el objetivo de mejorar la integración con otros niños que presentan TEA, y para facilitar el aprendizaje de las disciplinas. En esta fase, los requerimientos se obtuvieron en base a:

- Consulta con las partes interesadas (los niños con TEA, profesores, padres, psicólogos, fonoaudiólogos) a través de entrevistas.

- Revisión bibliográfica sobre el tema.

- Trabajo en terreno de niños con TEA, mediante observación participativa a través de un pilotaje, para aumentar las experiencias subjetivas de los observadores, durante todo el proceso.

- Focus Group con padres y/o apoderados de los niños seleccionados.

\subsection{Fase 2: Análisis}

Esta etapa se basó en las siguientes actividades (GARRETT, 2003):

- La exposición del problema principal que tiene que ser resuelto.

- Registro, perfeccionamiento de las ideas y selección de las más viables.

- Durante estas sesiones, se crearon los guiones gráficos o storyboards, utilizándolos para clarificar cómo se inserta el sistema en un escenario real.

\subsection{Fase 3: Diseño}

En esta etapa se desarrolló un conjunto de interfaces asociadas con los escenarios de uso de herramientas que reproducen el comportamiento real de los niños con TEA.

El prototipo se construyó principalmente en papel (SNYDER, 2003) y, después de aprobado por el equipo, se realizó el paso a una interfaz final, ver Figura 1. 

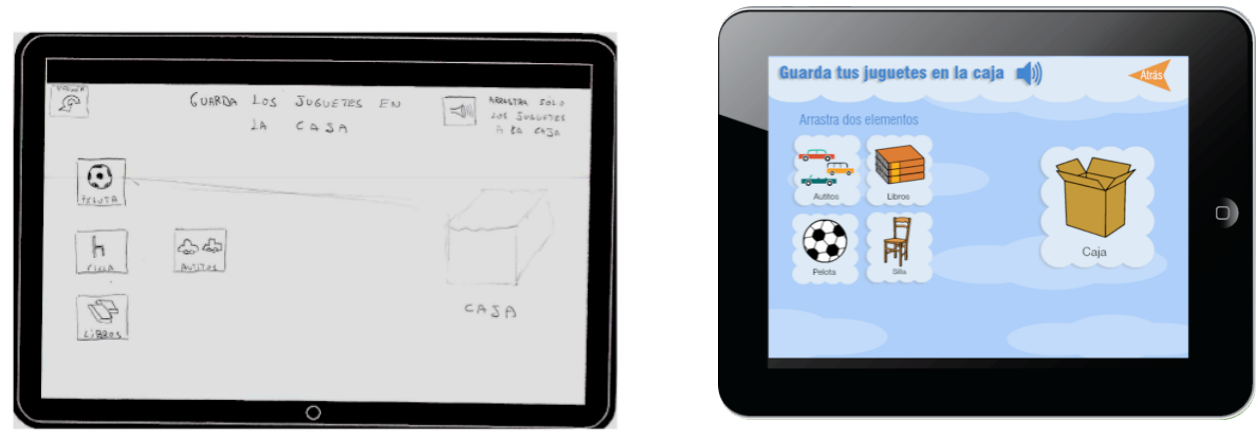

Figura 1. Ejemplo Prototipo en papel y digital de Proyect@Habilidades

\subsection{Fase 4: Evaluación}

La evaluación se realizó posterior al primer prototipo funcional. El objetivo fue conseguir que las personas involucradas en este proyecto (los niños con TEA, padres y terapeutas) utilizaran el producto, y así verificar la interacción entre los involucrados y la aplicación. El detalle de esta etapa es presentada en la Sección Validación de la Aplicación y Resultados Preliminares.

\section{Aplicación Propuesta}

La aplicación desarrollada se centra en el nivel 3 de la educación especial Chilena (DTO-170, 2010). Este reglamento define cinco niveles de actividades para mejorar las habilidades sociales y cognitivas de los niños con TEA. Un niño es diagnosticado inicialmente en un nivel específico. Los niños diagnosticados en el nivel 1 son los que tienen necesidades especiales más críticas.

La aplicación cuenta con 3 tipos de situaciones (casa, escuela, calle), cada una de ellas con 4 ó 5 tareas. La complejidad de las tareas en cada actividad aumenta gradualmente. Cada tarea presenta un problema específico asociado a la planificación (descrito por una voz en off y reforzada por una descripción textual). Para resolver el problema, el niño(a) debe ser capaz de organizar y elaborar un plan, cuando el plan es socialmente correcto, la interfaz presenta un refuerzo visual y audible positivo. Si el niño no toma ninguna acción, la instrucción se puede repetir. En la Figura 2, es presentada una de las interfaces finales de Proyect@ Habilidades, y en la tablas 1, 2 y 3 se presentan las actividades a realizar por cada tipo de situación (casa, escuela, calle).

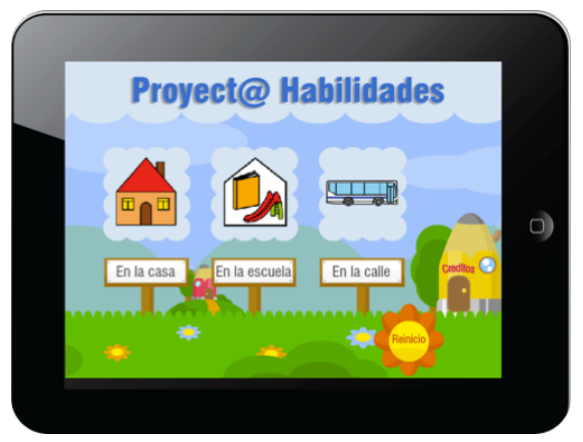

Figura 2. Interfaz Principal Proyect@Habilidades 
CBIE-LACLO 2015

Anais dos Workshops do IV Congresso Brasileiro de Informática na Educação (CBIE 2015)

Tabla 1. Situación En la Casa

\begin{tabular}{|l|l|}
\hline Tarea & Descripción \\
\hline 1 & Actividad que consiste en arrastrar el pictograma de un niño enfermo al hospital. \\
\hline 2 & $\begin{array}{l}\text { Actividad que consiste en arrastrar la ropa de vestir a los números que corresponda } \\
\text { según el orden para vestirse. }\end{array}$ \\
\hline 3 & $\begin{array}{l}\text { Actividad que consiste en arrastrar elementos donde corresponda (refrigerador y } \\
\text { microondas a la cocina, televisor y cama a la pieza). }\end{array}$ \\
\hline 4 & $\begin{array}{l}\text { Actividad que consiste en arrastrar } 3 \text { elementos necesarios para realizar un } \\
\text { cumpleaños (dulces, bebidas, torta). }\end{array}$ \\
\hline 5 & $\begin{array}{l}\text { Juego que consiste en arrastrar a un número cada una de las actividades para } \\
\text { organizar un fin de semana, esto según el gusto de cada usuario. }\end{array}$ \\
\hline
\end{tabular}

Tabla 2. Situación En la Escuela

\begin{tabular}{|l|l|}
\hline Tarea & Descripción \\
\hline 1 & Actividad que corresponde en arrastrar el pictograma manos sucias al baño. \\
\hline 2 & Actividad que corresponde en arrastrar los dos juguetes presentes a la caja. \\
\hline 3 & $\begin{array}{l}\text { Actividad que corresponde en arrastrar los pictogramas mochila y libro a la sala de } \\
\text { clases, y los pictogramas juguetes y pelota al patio. }\end{array}$ \\
\hline 4 & $\begin{array}{l}\text { Juego que consiste en arrastrar cada una de las actividades de los niños al número } \\
\text { correspondiente según el orden de llegada a la escuela (saludar, colgar mochila, } \\
\text { poner capa, trabajar). }\end{array}$ \\
\hline 5 & $\begin{array}{l}\text { Juego que consiste en arrastrar la actividad al número correspondiente para realizar } \\
\text { un almuerzo en orden (poner mesa, sentarse, comer, lavar dientes). }\end{array}$ \\
\hline
\end{tabular}

Tabla 3. Situación En la Calle

\begin{tabular}{|l|l|}
\hline Tarea & Descripción \\
\hline 1 & Actividad que corresponde en arrastrar los pasos para ir a un supermercado. \\
\hline 2 & Actividad que corresponde en establecer los pasos para pagar en un supermercado. \\
\hline 3 & Actividad que corresponde en arrastrar el elemento a un microbús vacío. \\
\hline 4 & Actividad que corresponde en arrastrar el elemento a la microbús lleno. \\
\hline
\end{tabular}

\section{Validación de la Aplicación y Resultados Preliminares}

En la figura 3, es presentada la estrategia de pruebas que se realizó a la aplicación.

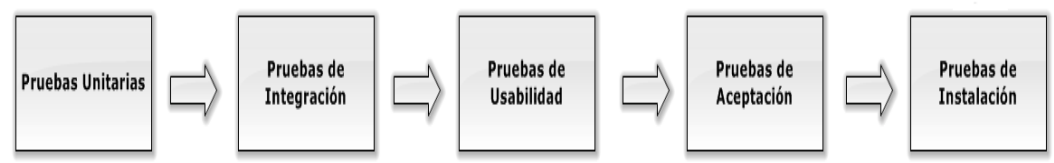

Figura 3. Interfaz Principal Proyect@Habilidades

Dentro de los tipos pruebas realizadas dentro del proyecto, unas de suma importancia fueron las de evaluación de usabilidad (CHILANA; WOBBROCK; KO, 2010). La evaluación de la usabilidad se divide generalmente entre métodos de inspección y prueba (DE KOCK; VAN BILJON; PRETORIUS, 2009). Dentro de los 
métodos de inspección, se decidió utilizar Evaluación Heurística, ya que este método permite encontrar los principales problemas y confeccionar un informe para mejorar la usabilidad de la aplicación (NIELSEN; MOLICH, 1990).

En la evaluación, fue realizada por 3 estudiantes de 6to año de Ingeniería Informática, todos ellos con una experiencia de al menos 1 año realizando evaluaciones de usabilidad. Se detectaron 12 potenciales errores, con una criticidad máxima de 4,33 y mínima de 1,32, con una desviación estándar de 0,95 , todo esto en una escala de 0 a 8 . Los principales potenciales errores fueron asociadas a las heurísticas visibilidad del estado del sistema, consistencia y estándares y prevención de errores. Estos potenciales errores fueron corregidos para posteriormente seguir en la ejecución de los métodos prueba.

En los métodos de prueba se decidió utilizar las "Pruebas con Usuarios", ya que permite obtener datos cuantitativos sobre el rendimiento de usuarios reales (niños con TEA) en la prueba. Para las pruebas con usuarios, 8 niños que se encontraban en los niveles 2 ó 3 (3 y 5 respectivamente) (DTO-170, DTO-815), ejecutaron, en una tablet de 7 pulgadas, todas las actividades propuestas en la aplicación. Cabe mencionar que todo esto fue posterior a la firma del consentimiento de parte de los padres de los niños con TEA. Por otra parte, se trabajó con niños del nivel 2 y 3 de acuerdo al decreto 815 y 170 de educación especial, el que menciona que estos niveles buscan trabajar las siguientes conductas (DTO-170, DTO-815):

- Nivel 2: Afianzar las conductas adaptativas que permitan a la persona un mejor nivel de vida y una mayor interacción con su medio ambiente.

- Nivel 3: Desarrollar habilidades y destrezas que permitan al educando desenvolverse en ambientes normalizadores.

Los resultados por cada grupo, son presentados en las tabla 4.

Tabla 4. Resultados Niños nivel 2 y 3

\begin{tabular}{|l|l|l|l|l|l|l|}
\hline \multirow{2}{*}{ Actividades } & \multicolumn{3}{|l|}{ Resultados Niños Nivel 3 } & \multicolumn{2}{l|}{ Resultados Niños Nivel 2} \\
\cline { 2 - 7 } & $\begin{array}{l}{ }^{\circ} \text { T. } \\
\text { Estimado }\end{array}$ & $\begin{array}{l}\text { o } \mathbf{T} . \\
\text { Promedio }\end{array}$ & $\begin{array}{l}\text { Desv. } \\
\text { Std. }\end{array}$ & $\begin{array}{l}{ }^{\circ} \mathbf{T} . \\
\text { Estimado }\end{array}$ & $\begin{array}{l}{ }^{\circ} \mathbf{T} . \\
\text { Promedio }\end{array}$ & $\begin{array}{l}\text { Desv. } \\
\text { Std. }\end{array}$ \\
\hline Casa & $4 \mathrm{~min}$. & $5.49 \mathrm{~min}$ & 0.53 & $4 \mathrm{~min}$. & $3.31 \mathrm{~min}$ & 0.36 \\
\hline Escuela & $4 \mathrm{~min}$. & $3.50 \mathrm{~min}$ & 0.84 & $4 \mathrm{~min}$. & $3.49 \mathrm{~min}$ & 0.77 \\
\hline Calle & $3 \mathrm{~min}$ & $2.36 \mathrm{~min}$ & 0.57 & $3 \mathrm{~min}$ & $1.55 \mathrm{~min}$ & 0.40 \\
\hline
\end{tabular}

De los resultados de la evaluación se puede mencionar que:

Los alumnos de nivel 3 demoran menos tiempo en realizar las actividades en todos los ámbitos y con menos errores que los de nivel 2. Conclusiones: Los niños de nivel 3 tienen un mejor manejo de actividades de organización y planificación, por esto realizan las actividades en menor tiempo y con menos errores que los de nivel 2.

2) Los niños de nivel 2 comenten muchos más errores en las actividades de casa. Conclusiones: Los niños de nivel 2 poseen un conocimiento de la organización y planificación en el colegio, sin embargo, en el ámbito de la casa tienen muchos problemas para identificar o realizar las actividades de manera correcta, por lo que la 
aplicación podría ser de mucha ayuda para dar ese soporte de trabajo a los profesionales y familiares.

Además a un grupo de expertos, después utilizar la aplicación, se les solicitó responder un instrumento basado en el estudio y evaluación de objetos de aprendizaje (Learning Object Evaluation Metric) (KAY; KNAACK, 2008). Este instrumento evalúa 4 puntos (Interactividad, Diseño, Compromiso y Usabilidad), los cuales fueron adaptados al Proyect@ Habilidades. Este instrumento está disponible en: ("Evaluación Proyect@ Habilidades Survey”, 2015). Esta evaluación fue respondida por 25 expertos, para esto se les realizaron unas preguntas sobre su perfil obteniendo los siguientes resultados: 11 fonoaudiólogos, 9 educadores especiales, 3 psicólogos y 2 terapeutas ocupacionales. De ellos 11 han trabajado más de 5 años con niños con TEA, otros 11 han trabajado entre 1 y 5 y los restantes 3 han trabajado menos de un año con niños con TEA. En la evaluación se esperó que ningún punto de la evaluación tenga un porcentaje mayor a $50 \%$ en la categoría deficiente. De las Tablas 6 a 9, se presentan los resultados obtenidos en los ámbitos de Interactividad, Diseño, Compromiso y Usabilidad. La evaluación con calificación excelente corresponde a un aspecto muy positivo, y el con una calificación deficiente, significará un aspecto a mejorar a la brevedad.

Tabla 6. Resultados Interactividad

\begin{tabular}{|l|l|l|l|}
\hline Pregunta Interactividad & Excelente & Aceptable & Deficiente \\
\hline 1 Interacciones significativas & $83.33 \%$ & $8.33 \%$ & $8.33 \%$ \\
\hline 2 Control global & $73.83 \%$ & $25.07 \%$ & $1.1 \%$ \\
\hline $\begin{array}{l}3 \text { Contenido multimedia añade valor al } \\
\text { aprendizaje }\end{array}$ & $66.67 \%$ & $20.83 \%$ & $12.5 \%$ \\
\hline
\end{tabular}

Tabla 7. Resultados Diseño

\begin{tabular}{|l|l|l|l|}
\hline Pregunta Diseño & Excelente & Aceptable & Deficiente \\
\hline 1 Consistencia & $60.00 \%$ & $40.00 \%$ & $0.00 \%$ \\
\hline 2 Etapas & $95.24 \%$ & $4.76 \%$ & $0.00 \%$ \\
\hline 3 Etiquetado & $71.43 \%$ & $28.57 \%$ & $0.00 \%$ \\
\hline 4 Legibilidad & $82.55 \%$ & $17.45 \%$ & $0.00 \%$ \\
\hline
\end{tabular}

Tabla 8. Resultados Compromiso

\begin{tabular}{|l|l|l|l|}
\hline Pregunta Compromiso & Excelente & Aceptable & Deficiente \\
\hline 1 Calidad de la evaluación & $60.00 \%$ & $40.00 \%$ & $0.00 \%$ \\
\hline 2 Atractivo (aspecto) & $60.00 \%$ & $40.00 \%$ & $0.00 \%$ \\
\hline 3 Gráficos & $66.67 \%$ & $33.33 \%$ & $0.00 \%$ \\
\hline 4 Modo de aprendizaje & $54.16 \%$ & $45.84 \%$ & $0.00 \%$ \\
\hline 5 Motivación & $80.95 \%$ & $19.05 \%$ & $0.00 \%$ \\
\hline
\end{tabular}

Tabla 9. Resultados Usabilidad

\begin{tabular}{|l|l|l|l|}
\hline Pregunta Usabilidad & Excelente & Aceptable & Deficiente \\
\hline 1 Naturalidad de uso & $85.71 \%$ & $14.29 \%$ & $0.00 \%$ \\
\hline
\end{tabular}


CBIE-LACLO 2015

Anais dos Workshops do IV Congresso Brasileiro de Informática na Educação (CBIE 2015)

\begin{tabular}{|l|l|l|l|}
\hline 2 Orientación & $76.19 \%$ & $23.81 \%$ & $0.00 \%$ \\
\hline 3 Navegación & $71.43 \%$ & $23.81 \%$ & $4.76 \%$ \\
\hline 4 Instrucciones & $90.48 \%$ & $4.76 \%$ & $4.76 \%$ \\
\hline 5 Nivel de idiomas adecuado & $71.43 \%$ & $28.57 \%$ & $0.00 \%$ \\
\hline
\end{tabular}

Las conclusiones de los resultados de la evaluación experta nos dejan los siguientes análisis:

En cuanto a Interactividad es posible mencionar que en los tres puntos existe una buena aprobación por parte de los profesionales, siendo el ámbito mejor evaluado el relacionado con la consistencia que obtiene el $83.33 \%$ de las personas que creen que las interacciones del sistema son significativas. Esto permite decir que en este ámbito los profesionales creen que la aplicación si entrega interacciones significativas y que permitirán el aprendizaje a los niños con TEA al trabajar en el futuro con ella.

En cuanto al Diseño es posible visualizar ver que la aprobación es alentadora, pues ninguno de los profesionales marcó el ítem deficiente de la evaluación. En relación al punto de mayor aprobación, es el relacionado con las etapas, lo que lleva a concluir que se logró un buen trabajo en cuanto a la distribución de estas, no son desordenadas y se entiende perfectamente lo que en ellas se quiso plasmar.

En relación al Compromiso, la aprobación también muy positiva, pues al igual que en la tabla de interactividad, ninguno de los profesionales marco el ítem deficiente de la evaluación. El ámbito mejor evaluado, es el relacionado con la motivación entregada por Proyect@ Habilidades, llegando al 80.95\%. Esto nos dice que aprender las tareas de la aplicación son muy motivadoras y atractivas para los niños y eso queda reafirmado por la opinión de los profesionales que trabajan con ellos.

En cuanto a la Usabilidad de la aplicación también se obtuvieron buenos resultados pues en todos los puntos se supera al $71 \%$ en el ítem excelente, lo que es la máxima aprobación. La mejor evaluada, es la relacionada con las instrucciones que se entregan en la aplicación. Esto para la aplicación es muy importante, ya que las instrucciones deben ser explícitas para no confundir a los niños cuando realicen las actividades.

Para finalizar la aplicación al 2 de agosto del 2015, posee 11.505 instalaciones, distribuyéndose principalmente en los países de España (41,62\% - 4.345), Chile $(13,40 \%$ - 1.399), México (12,16\% - 1.269) y Argentina (12,03\% - 1.186).

\section{Conclusiones y Trabajo Futuro}

En Chile se torna de gran importancia desarrollar sistemas que cooperen con personas con necesidades especiales. Esto debido a la falta de este tipo de desarrollos a nivel nacional, es por esto que la aplicación resultante es un aporte para dar soporte a los profesionales y familias de niños/as con TEA. Esto quedó demostrado con los comentarios recibidos y con las evaluaciones que se obtuvieron en las distintas pruebas realizadas.

Se lograron cubrir todos los objetivos planteados en ellos, y se logró desarrollar una aplicación que ayuda a fomentar las funciones ejecutivas (organización y planificación) en niños y niñas con TEA. Esto fue posible gracias a que se lograron 


\section{CBIE-LACLO 2015}

Anais dos Workshops do IV Congresso Brasileiro de Informática na Educação (CBIE 2015)

determinar las estrategias mayormente utilizadas por los terapeutas en Chile, luego esas estrategias fueron llevadas a una aplicación para dispositivos multitouch, la cual se puso a disposición en Google Play. Finalmente se determinó el impacto de la aplicación en cuanto al apoyo al terapeuta, mediante una serie de pruebas tanto a usuarios finales del sistema, como a los profesionales que trabajarán con Proyect@ Habilidades a futuro.

En cuanto a trabajos futuros, la aplicación puede seguir mejorando en muchos ámbitos, por ejemplo se pueden crear nuevos juegos que potencien las funciones ejecutivas que no fueron abordadas y hacer etapas más complejas de las que existen en la actualidad. Además se pueden generar más actividades en Otros Ámbitos, pues existen muchas tareas de la vida diaria que pueden ser incluidas en ese aspecto.

\section{Agradecimientos}

Roberto Muñoz es beneficiario de la Beca de Doctorado INF-PUCV 2015.

Agradecemos a la Escuela Especial Germina y a todos los involucrados en el proyecto.

\section{Referencias}

ABUZZ. Niño juego de memoria Alimento. Disponível em: $<$ https://play.google.com/store/apps/details?id=com.iabuzz.MemoryMatchFood>.

Acesso em: 1 jun. 2015.

AITKEN, K. S. Bölte and J. Hallmayer (Eds.): Autism Spectrum Conditions: FAQs on Autism, Asperger Syndrome, and Atypical Autism Answered by International Experts. Journal of Autism and Developmental Disorders, v. 42, n. 9, p. 2023-2024, 2011.

BEREZNAK, S. et al. Video Self-Prompting and Mobile Technology to Increase Daily Living and Vocational Independence for Students with Autism Spectrum Disorders. Journal of Developmental and Physical Disabilities, v. 24, n. 3, p. 269-285. 2012.

CHILANA, P. K.; WOBBROCK, J. O.; KO, A. J. Understanding usability practices in complex domains: CHI '10. New York, NY, USA: ACM, 2010.

COCKBURN, J. et al. SmileMaze: A tutoring system in real-time facial expression perception and production in children with Autism Spectrum Disorder.Proceedings from the 8th IEEE International Conference on Automatic Face \& Gesture Recognition. IEEE, 2008

DE KOCK, E.; VAN BILJON, J.; PRETORIUS, M. Usability evaluation methods: mind the gaps. New York, NY, USA: ACM, 2009

DIGENNARO REED, F. D.; HYMAN, S. R.; HIRST, J. M. Applications of technology to teach social skills to children with autism. Research in Autism Spectrum Disorders, v. 5, n. 3, p. 1003-1010, 2011.

DTO-170 21-ABR-2010 MINISTERIO DE EDUCACIÓN - Ley Chile - Biblioteca del Congreso Nacional. Disponível em: <http://www.leychile.cl/Navegar?idNorma=1012570>. Acesso em: 19 jun. 2014.

DTO-815 07-OCT-1991 MINISTERIO DE EDUCACIÓN. Disponível em: $<$ http://www.leychile.cl/Navegar?idNorma $=226792 \& i d P a r t e=0>$. Acesso em: 9 abr. 2013. 


\section{CBIE-LACLO 2015}

Anais dos Workshops do IV Congresso Brasileiro de Informática na Educação (CBIE 2015)

ESCOBEDO, L. et al. MOSOCO: a mobile assistive tool to support children with autism practicing social skills in real-life situations. Proceedings of the 2012 ACM annual conference on Human Factors in Computing Systems. USA: ACM, 2012.

Evaluación Proyect@ Habilidades Survey. Disponível em: <https://goo.gl/LDMsBf>. Acesso em: 1 jun. 2015.

GARRETT, J. J. The Elements of User Experience: User-Centered Design for the Web. American Institute of Graphic Arts, 2003.

HOURCADE, J. P. et al. Evaluation of tablet apps to encourage social interaction in children with autism spectrum disorders. ACM Press, 2013.

KAY, R.; KNAACK, L. A multi-component model for assessing learning objects: The learning object evaluation metric (LOEM). Australasian Journal of Educational Technology. v. 24, n. 5, p. 574-591, 2008.

LOZANO, J.; BALLESTA, J.; ALCARAZ, S. Software para ensenar emociones al alumnado con trastornos del espectro autista. Revista Científica de Educomunicación, v. 18, n. 36, p. 139-148, 2011.

MECHLING, L. C.; GAST, D. L.; SEID, N. H. Using a Personal Digital Assistant to Increase Independent Task Completion by Students with Autism Spectrum Disorder. Journal of Autism and Developmental Disorders, v. 39, n. 10, p. 1420-1434, 2009.

MEGÍAS, M. G. et al. Sc@ut: Platform for Communication in Ubiquitous and Adaptive Environments Applied for Children with Autism. 8th ERCIM Workshop on User Interfaces for All, Vienna, Austria, 2004.

MUNOZ, R. et al. Development of Software that Supports the Improvement of the Empathy in Children with Autism Spectrum Disorder. nov. 2012 : IEEE, nov. 2012. p. 223-228.

NIELSEN, J.; MOLICH, R. Heuristic evaluation of user interfaces. ACM Press, 1990

OZONOFF, S. et al. Executive Function Abilities in Autism and Tourette Syndrome: An Information Processing Approach. Journal of Child Psychology and Psychiatry, v. 35, n. 6, p. 1015-1032, set. 1994.

Proyect@Habilidades - Aplicaciones de Android en Google Play. Disponível em: $<$ https://play.google.com/store/apps/details?id=air.Habilidades $>$. Acesso em: 29 maio. 2015.

SNYDER, C. Paper prototyping: the fast and easy way to design and refine user interfaces. San Francisco, CA: Morgan Kaufmann Publishers, 2003.

VAN DEURZEN, L. et al. Theory of mind and executive functioning in autism. European Psychiatry, v. 23, p. S401. 2008.

VAN DIJK, D. et al. Users as Designers A hands-on approach to Creative Research. Waag Society, 2011.

VENKATESH, S. et al. Pervasive multimedia for autism intervention. Pervasive and Mobile Computing, v. 8, n. 6, p. 863-882, dez. 2012.

VOLKMAR, F. R.; REICHOW, B.; WIESNER, L. A. Autism and Aspergers. In: Encyclopedia of Adolescence. Elsevier, 2011.p. 62-67. 2011. 